\title{
On the Mechanism of the Hofmeister Effect
}

Marc C. Gurau, Soon-Mi Lim, Edward T. Castellana, Fernando Albertorio, Sho Kataoka and Paul S. Cremer*

Department of Chemistry, Texas A\&M University, P.O. Box 30012, College Station,

Texas 77843 Tel : 979-862-1200, Fax : 979-845-7561

\section{Supplemental Information:}

\section{Vibrational Sum Frequency Spectroscopic Measurements}

The visible and infrared inputs for all experiments were generated by an optical parametric generator/amplifier (OPG/OPA from Laser Vision, Bellevue, WA) pumped with a Nd:YAG laser (Continuum, PY 61 custom, Santa Clara, CA). The laser generated a $50 \mathrm{~mJ}, 17 \mathrm{ps}$ pulse at $1064 \mathrm{~nm}$. Within the OPG/OPA a KTP crystal was used to double a portion of the fundamental to create $532 \mathrm{~nm}$ radiation. In addition to its use in subsequent wave mixing processes to generate tunable infrared, $1 \mathrm{~mJ}$ of green intensity was used as the visible source in the sum-frequency experiments. The remaining $532 \mathrm{~nm}$ beam underwent a parametric process in two KTP crystals before a final difference frequency generation (DFG) process through two KTA crystals. The mid-IR input was generated by mixing the idler from the parametric process and a portion of the $1064 \mathrm{~nm}$ fundamental. The result of the parametric and DFG processes was an infrared beam whose frequency could be tuned between $2000 \mathrm{~cm}^{-1}$ and $4000 \mathrm{~cm}^{-1}$ by adjusting the angles of the various crystals.

The spectra reported in Figures $1 \& 2$ have each been normalized to the nonresonant response from a piece of crystalline quartz (Z-cut). The quartz disk was placed in the beam path with its top surface at the same position as the air/water interface, as judged by the path of the reflected visible beam. After background subtraction all spectra were divided by the non-resonant response of the quartz sample to correct for changes in alignment and infrared intensity that occurred over the frequency range investigated. The normalized spectra from the $\mathrm{D}_{2} \mathrm{O}$ samples were then fit to a Lorentzian model using 
Matlab software. The $v_{\mathrm{s}}\left(\mathrm{CH}_{3}\right) / v_{\mathrm{s}}\left(\mathrm{CH}_{2}\right)$ ratios reported in Table 1 were obtained by dividing the oscillator strength for the methyl symmetric stretch feature by that of the methylene symmetric stretch feature

\section{$\underline{\text { Trough and Monolayer Preparation }}$}

The visible and infrared lasers were spatially and temporally aligned to the air/water interface of a Langmuir trough (Model 601M, Nima, UK). When aligned at the surface, the visible and infrared beams generated a polarization that acted as the source for the sum frequency signal. The signal was then directed to a photomultiplier tube where its intensity could be recorded on a computer. The same computer was also used to control the positions of the crystals in the OPG/OPA allowing for automation of the signal collection process through a program written in Labview.

The salt solution in the Langmuir trough was held at a constant temperature of $20^{\circ} \mathrm{C}$ by the use of a water recirculator to flow anti-freeze through the hollow Teflon body. The Langmuir trough's two barriers moved across the water's surface to control the area occupied by the monolayer. The surface pressure of the monolayer was monitored with a Wilhelmy plate made from chromatographic paper.

Salt solutions (10 $\mathrm{mM}$ concentration) were made by dissolving the appropriate amount of a sodium salt (obtained from Aldrich and used without purification) into either $\mathrm{D}_{2} \mathrm{O}$ (Cambridge Isotope Labs) or $\mathrm{H}_{2} \mathrm{O}$ (Resistivity > $18 \mathrm{M} \Omega$, Barnstead NANOpure filtration system, Dubuque, IA). For each experiment a constant volume of salt solution $(50 \mathrm{~mL})$ was delivered to the trough via a volumetric pipette. The monolayers were prepared by touching drops of an octadecylamine solution (ODA $0.25 \mathrm{mg} / \mathrm{mL}$ in $\mathrm{CHCl}_{3}$ ) to the water's surface using a $25 \mu \mathrm{L}$ syringe. ODA was added to the surface until the surface pressure exceeded $20 \mathrm{mN} / \mathrm{m}$. The barriers were then set to continuously adjust the area occupied by the monolayer so as to maintain a surface pressure of $15 \mathrm{mN} / \mathrm{m}$ for the duration of the spectroscopic experiments. At the conclusion of each experiment the monolayer and subphase were removed with an aspirator. The trough was then rinsed several times with purified water before the addition of a new subphase. 


\section{Choice of Subphases}

Recent investigations into Hofmeister effects have demonstrated that better agreement between theory and experiment can be achieved by including dispersion effects into the theoretical models. In fact, at the higher ionic strength concentrations typical of Hofmeister investigations, theory predicts that attractive dispersion forces can even dominate the repulsive electrostatic forces. Such effects are directly related to the polarizibility of the ion under investigation. Larger ions with lower overall charge densities, such as polyatomic anions, will be the most susceptible to dispersion forces whereas divalent ions and most cations are less likely to be affected due to the higher charge densities experienced in these systems. These properties are of particular interest when examining different effects that such ions will have on an amphiphilic monolayer. In this case theory suggests that softer ions will more strongly interact with the alkyl portion of the monolayer through dispersion forces, allowing them to penetrate further past the headgroups.

It was a goal of this work to collect data from samples where the effects of each individual anion could be observed separately. To this end, all solutions were prepared by the addition of the pure salt to purified water. While it would have been possible to precisely control the $\mathrm{pH}$ of these solutions through the addition of buffers, this would have necessitated the addition of a mixture of ions that would have complicated the interpretation of the experiments. In order to still maintain nearly constant $\mathrm{pH}$ values, we elected to use only the corresponding salts of strong acids; hence, sodium phosphate and sodium fluoride were not employed as they would have increased the $\mathrm{pH}$. The salt of the weakest acid used was $\mathrm{NaSCN}\left(\mathrm{pK}_{\mathrm{a}}=0.9\right.$ for $\left.\mathrm{HSCN}\right)$ and everything else was even stronger. The $\mathrm{pH}$ value of the purified water was 5.6. This value presumably represents the presence of trace carbonic acid that is typically found in purified water that is in contact with ambient air that contains $\mathrm{CO}_{2}$. The addition of $10 \mathrm{mM}$ salt to the water yielded $\mathrm{pH}$ values that ranged from 5.6 to 6.0 in all cases.

It should be noted that all the salt solutions had the identical ionic strength with the exception of the $\mathrm{Na}_{2} \mathrm{SO}_{4}$ solution, which had a value three times higher than the others because of the divalent nature of the anion. Control experiments with a third of the $\mathrm{Na}_{2} \mathrm{SO}_{4}$ concentration showed that the higher ionic strength value did not change the 
ordering of any of the results with respect to the other ions. In other words, a choice of constant ionic strength or constant concentration gave rise to the same conclusions for this system.

\section{$\underline{\text { Surface Potential Measurements }}$}

The surface potentials of the ODA monolayers on the various subphases were measured using a separate Langmuir trough (KSV 5000) that was equipped with a vibrating capacitor surface potential meter (Kelvin probe). In these experiments, subphases were again prepared by dissolving the appropriate amount of salt in $\mathrm{H}_{2} \mathrm{O}$. The trough was cleaned with chloroform and pure water before each experiment. A freshly prepared subphase solution was then added to the trough and its surface pressure and surface potential were monitored for approximately 15 minutes before spreading the monolayer. The pressure and potential were zeroed at the end of this time so that all subsequent pressure and potential measurements were made relative to the bare subphase/air interface.

All monolayers were prepared by spreading $350 \mu \mathrm{L}$ of the same ODA solution used in the spectroscopic experiments. After allowing the spreading solvent to evaporate ( $\sim 15$ minutes) the barriers were compressed at a constant rate $(5 \mathrm{~mm} / \mathrm{min})$ until the surface pressure reached $15 \mathrm{mN} / \mathrm{m}$. The monolayer was then held at constant pressure for 30 minutes. The surface potential and pressure of each monolayer system was monitored throughout the entire experiment. The values reported in Table 1 represent an average obtained for each monolayer after the system reached its final pressure of 15 $\mathrm{mN} / \mathrm{m}$.

\section{Peak Fitting for the $\mathrm{OH}$ Stretch Region in Figure 2}

It can be seen qualitatively in Figure 2 that the $\mathrm{OH}$ stretch intensities from the near surface water at the ODA/aqueous interface do not follow the Hofmeister series nearly as closely the $v_{\mathrm{s}}\left(\mathrm{CH}_{3}\right) / v_{\mathrm{s}}\left(\mathrm{CH}_{2}\right)$ ratios or the surface potential measurements do. Here we provide the quantitative values of the oscillator strengths for the two main water peaks using a Voigt profile to fit the data. This analysis reveals that the lower frequency peak is approximately $3205 \mathrm{~cm}^{-1}$, while the higher frequency peak is near $3425 \mathrm{~cm}^{-1}$. 
These frequencies vary only slightly through the series of ions tested. The oscillator strengths (in arbitrary units) are given in the table. The peak intensities from the sulfate system were so weak that we were unable to fit the data and, hence, report oscillator strengths of zero for this anion.

\begin{tabular}{|c|c|c|c|c|c|c|c|}
\hline Peaks & $\mathrm{NaCl}$ & $\mathrm{NaBr}$ & $\mathrm{NaI}$ & $\mathrm{NaNO}_{3}$ & $\mathrm{NaSCN}$ & $\mathrm{NaClO}_{4}$ & $\mathrm{Na}_{2} \mathrm{SO}_{4}$ \\
\hline $3205 \mathrm{~cm}^{-1}$ & 441 & 419 & 443 & 442 & 296 & 586 & 0 \\
\hline $3425 \mathrm{~cm}^{-1}$ & 185 & 178 & 129 & 103 & 117 & 228 & 0 \\
\hline
\end{tabular}

